\title{
Consideraciones sobre el ensayo de ANSTETT y el comportamiento de los cementos frente a sulfatos
}

\author{
Prof. Dr. JOSE CALLEJA \\ IETCC \\ Licdo. MANUEL AGUANEII \\ CeMentos porthand VALDERRIBAS, S. $A$.
}

\section{R E S U M E N}

Se ponen de relieve aspectos aparentemente anómalos, relativos a cementos portland de alta resistencia, cementos portland resistentes al yeso y cementos puzolánicos, en cuanto a su comportamiento en el laboratorio frente al método de ensayo de ANSTETT, y en la práctica frente a la acción del yeso y del sulfato magnésico.

Se trata de explicar las virtuales anomalías, considerando las influencias de los factores físicos y químicos inherentes a los materiales y a los métodos utilizados.

Se concluye llamanda la atención sobre la dudosa fiabilidad de algunos métodos para enjuiciar el comportamiento de determinados cementos, y sobre el peligro de establecer recomendaciones basadas en resultados incorrectamente interpretados y valorados.

\section{INTRODUCCION}

Se suelen recomendar — con fundamento— los cementos puzolánicos para hormigones que hayan de estar sometidos a ataques por medios agresivos sulfatados, con preferencia a otros cementos, entre ellos los portland ordinarios y de altas resistencias.

Los medios agresivos sulfatados tanto en el laboratorio como en la realidad, pueden ser de distinta naturaleza. Desde la más simple — sulfato sódico, o cálcico, o magnésico, solos en cada caso-, hasta la más compleja - agua de mar-, pasando por la intermedia de mezclas binarias de sulfatos — sulfatos cálcico y magnésico de determinados terrenos-.

En cada caso el mecanismo —o los varios mecanismos posibles- de la agresión pueden ser distintos en extensión y en intensidad. En consecuencia, los efectos son también diversos y se pueden manifestar de modo diferente, según sea la naturaleza del cemento contenido en el hormigón sometido al agresivo. En particular, si el cemento es puzolánico, ello dependerá también del tipo de la puzolana y del clínker, de la proporción de mezcla de ambos, de la finura de las partes y del conjunto, y de la curva granulométrica de éste. 
En cuanto al propio hormigón, los efectos del ataque por sulfatos, para un mismo tipo de cemento, dependerán de la compacidad - porosidad, permeabilidad, capilaridad_, la cuai depende a su vez de la dosificación de cemento, de la relación agua/cemento, de la naturaleza y granulometría de los áridos y de la preparación y curado del hormigón.

Entre los ensayos de laboratorio para determinar la durabilidad o resistencia química de los cementos frente a los sulfatos, los hay de la más variada naturaleza y condición en cuanto a los materiales del ensayo - pastas puras, morteros u hormigones-; en cuanto a las probetas - por su forma, tamaño, composición, confección, curado y tratamiento-; en cuanto a los medios agresivos - por su naturaleza, composición, concentración y forma de actuar-; por el caracter más o menos drástico de los propios métodos, etc.

En relación con tan variada panorámica no es extraño que ensayos diferentes den también resultados diferentes en cuanto al enjuiciamiento de un cemento determinado, y sobre todo en comparación con otros cementos de distinto tipo. Tampoco es extraño que los resultados de algunos de estos ensayos comparativos de laboratorio no se correspondan con los que se observan en el comportamiento real de los cementos en la práctica, frente a medios agresivos naturales. $\mathrm{Y}$ tampoco choca que, en la propia práctica real, hormigones con cementos menos adecuados se comporten mejor que otros con cementos más idóneos y al contrario. Pero todo ello, ¿al cabo de cuánto tiempo de observación? Porque, en función de dicho tiempo, también es posible que lo observado durante un período relativamente corto sea contrario a lo observado durante un periodo relativamente largo, y viceversa.

De todo ello se deduce que a veces se dan situaciones de duda o de perplejidad, cuando por tales faltas de correlación se observan resultados inesperados o en apariencia no razonables. En tales casos es muy fácil atribuir semejantes resultados a causas que no son operantes -o que lo son de forma muy secundaria-, frente a otras principales que se ig. noran o pasan desapercibidas, o a las que no se les da su verdadero valor.

Con esto se puede correr el riesgo de adoptar decisiones poco acertadas, ofreciendo con ello soluciones erróneas a determinados problemas de durabilidad. Esto es malo de por sí en casos aislados, pero es aún mucho peor cuando se trata de extrapolar, generalizar y publicar tales decisiones y tales soluciones en normas o, simplemente, en recomendaciones de utilización de unos u otros cementos para unas u otras finalidades relac:onadas con la durabilidad del hormigón (1).

\section{EL ENSAYO DE ANSTETT}

Sin entrar en su descripción detallada, ya que se da por bien conocido, el ensayo de ANSTETT, que pasa por ser uno de los más drásticos, actúa sobre pasta hidratada de cemento mezclada con yeso dihidrato y compactada bajo presión en una probeta, la cual se somete a la acción del agua que penetra en ella por succión y absorción capilar. Se supone que el deterioro consiste en la destrucción de la probeta por formación expansiva de ettringita, según el mecanismo general:

$$
\mathrm{C}_{x} \mathrm{AH}_{y}+3 \mathrm{CS}+(28+x-y) \mathrm{H} \rightarrow(x-3) \mathrm{CH}+\mathrm{C}_{3:} \mathrm{A}(\mathrm{C} \overline{\mathrm{S}})_{33} \mathrm{H}_{31}
$$

\subsection{El esayo de Anstett y los cementos puzolánicos}

En primer lugar, el ensayo de ANSTETT exige la hidratación previa del cemento, la cual, 
en unas condiciones y en un tiempo dados, puede ser fácil de conseguir en el caso de los cementos portland, y no tan fácil -incluso imposible- de lograr en el caso de los cementos puzolánicos.

La consolidación —endurecimiento- de éstos se produce en dos etapas, másł sucesivas que simultáneas. En la primera de ellas se hidrata la fracción de clínker, como en el caso del cemento portland. En la segunda tiene lugar la reacción entre la puzolana y la cal de hidrólisis liberada en la primera etapa. Esta etapa primera es relativamente rápida, mientras que la segunda es diferida y más lenta.

En el tiempo y en las condiciones de hidratación del cemento en el ensayo, si el portland se hidrata en un cien por ciento, el cemento puzolánico comparativamente se hidrata sólo en un setenta por ciento, supuesto que tal sea el porcentaje en peso de la fracción de clínker que contiene. Por consiguiente, en dicho tiempo la pasta del cemento puzolánico está menos consolidada —endurecida y resistente- que la del cemento portland.

En segundo lugar, al triturar y pulverizar la pasta hidratada y mezclarla con yeso se interrumpen -o por lo menos se perturban - las acciones puzolánicas que ya estuvieran en curso o incluso acabadas, y se dificultan las que en adelante pudieran transcurrir en otras condiciones más favorables, pues el yeso interpuesto puede entorpecer o reducir el contacto entre la cal y la puzolana, necesario para la reacción de fijación de la cal.

Por todo ello, las probetas mezcla de pasta y yeso prensadas y compactadas tienen menos resistencia en el caso del cemento puzolánico que en el caso del cemento portland, si a ambos cementos se les aplica el método de ANSTETT del mismo modo.

En tercer lugar, una pasta de tres componentes — clínker hidratado, puzolana y yesotiene más probabilidades de ser heterogénea que una de dos componentes - clínker hidratado y yeso-, y, por lo tanto, puede ser más fácilmente penetrable por el agua (por succión capilar), a pesar de ser sometidas ambas a la misma presión de compactación. El papel decisivo del agua en la formación expansiva de la ettringita es bien conocido.

$\mathrm{Si}$, además, la puzolana no es notoriamente más blanda que el clínker, al moler ambos conjuntamente se obtiene un cemento "más agrio", el cual puede no dar pastas tan plásticas como las de un cemento puzolánico con una puzolana más blanda, o incluso como las de un cemento portland ordinario, o de alta resistencia, finamente molidos. Esto puede contribuir también a una mayor y más rápida absorción de agua por succión capilar, en el caso de las pastas del cemento puzolánico con puzolana no demasiado blanda.

Por otra parte, el contacto entre yeso y pasta hidratada para formar ettringita expansiva, más fácil, más amplio y! más regular, homogéneo y "simétrico" en el caso del cemento portland, es más localizado y circunscrito a zonas y puntos en el caso del cemento puzolánico, pero por ello mismo puede dar lugar, en conjunto, a menor número de acciones expansivas menos extensas pero más intensas, y por ello mismo más destructivas.

Por todo lo cual, los cementos puzolánicos, en general, tienen bastantes probabilidades de ser más susceptibles que los portland frente al ensayo de ANSTETT. O, dicho de otro modo, el ensayo de ANSTETT es discriminatorio, en el sentido de que "trata peor" a los cementos puzolánicos que a los portland, o mejor a éstos que a aquéllos. Algo parecido se podría decir, en principio, de los demás cementos con otras adiciones o componentes secundarios — cenizas volantes y escorias siderúrgicas-, así como de algunos otros métodos de ensayo severos, como el ASTM. 


\subsubsection{Consecuencias}

Si se utiliza el método de ANSTETT para comparar el comportamiento en el laboratorio de cementos de distinto tipo, es posible que los que mejor se comporten sean los de tipo portland, frente a otros -y en particular frente a los puzolánicos-. A ello responde la parte superior del esquema gráfico.

Sin embargo, si se utilizan otros métodos (WITTEKINDT, MERRIMAN, KOCH \& STEINEGGER, etc.), los resultados se invierten, situándose más en consonancia con el comportamiento esperado y real de unos y otros cementos en la práctica. De esto hay suficientes pruebas experimentales, de las cuales se ha dado cuenta, con aportaciones de datos concluyentes, en diversos lugares y ocasiones (2) (3) (4) (5).

\subsection{El ensayo de Anstett y los cementos portland}

Entre los cementos portland se pueden distinguir, a estos efectos, dos tipos extremos -que serían los tipos III y V ASTM-: los de alta saturación de cal, ricos en $\mathrm{C}_{3} \mathrm{~S}$ y relativa y circunstancialmente en $\mathrm{C}_{3} \mathrm{~A}$, con altas resistencias, de endurecimiento rápido y gran calor de hidratación, y los de características contrarias, especialmente por lo que se refiere al contenido de $\mathrm{C}_{3} \mathrm{~A}$.

Frente al ensayo de ANSTETT e incluso al ASTM cabe esperar, lógicamente, que los segundos se comporten mucho mejor que los primeros, y así se ha podido observar y confirmar una vez más recientemente, en un caso particular (6). Véase el esquema.

La explicación bien conocida del hecho reside en factores químicos más que en físicos. En efecto, un cemento V ASTM P-350-Y según normas españolas (7), tiene un contenido mucho menor de aluminatos cálcicos anhidros, causantes de la formación expansiva de ettringita, a través de la reacción de sus productos de hidratación con los sulfatos.

A este hecho, correlativo de un contenido más elevado de aluminatos en los cementos III ASTM (P-ARI) según las normas españolas (7), hay que añadir el de que éstos dan pastas más ricas en cal de hidrólisis, lo cual facilita la formación de más abundante ettringita expansiva.

Frente a estos factores de cáracter químico, los de naturaleza física, como puede ser una mayor resistencia mecánica a toda edad de los cementos III frente a los de tipo V por razón de su más rápido endurecimiento, o una posible mayor compacidad -menor porosidad, permeabilidad, penetrabilidad y succión capilar - de las probetas ANSTETT del cemento III que haga más difícil y lenta la penetración de agua en ellas, cuentan bastante menos.

La posible mayor compacidad de las probetas ANSTETT de un cemento III con relación a las de un cemento $\mathrm{V}$, podría ser debida a una mayor plasticidad de la mezcla amasada de yeso y pasta hidratada, la cual al ser sometida a la presión de compactación del ensayo, "se cerraría" más. Lo cual, a su vez, puede obedecer a una finura inicial bastante mayor del cemento III, cuando su condición de alta resistencia inicial se consigue exclusivamente o principalmente forzando en demasía la finura de su clínker. Finura mayor que también contribuye a proporcionar en un tiempo dado un grado más avanzado de hidratación y, por lo tanto, una mayor consolidación —resistencia mecánica-.

Estas consideraciones de carácter físico cuentan en cambio mucho más, cuando se trata de explicar un comportamiento mejor de los cementos de tipo III ASTM en relación con los cementos puzolánicos frente al ensayo de ANSTETT y al propio ensayo ASTIM. 


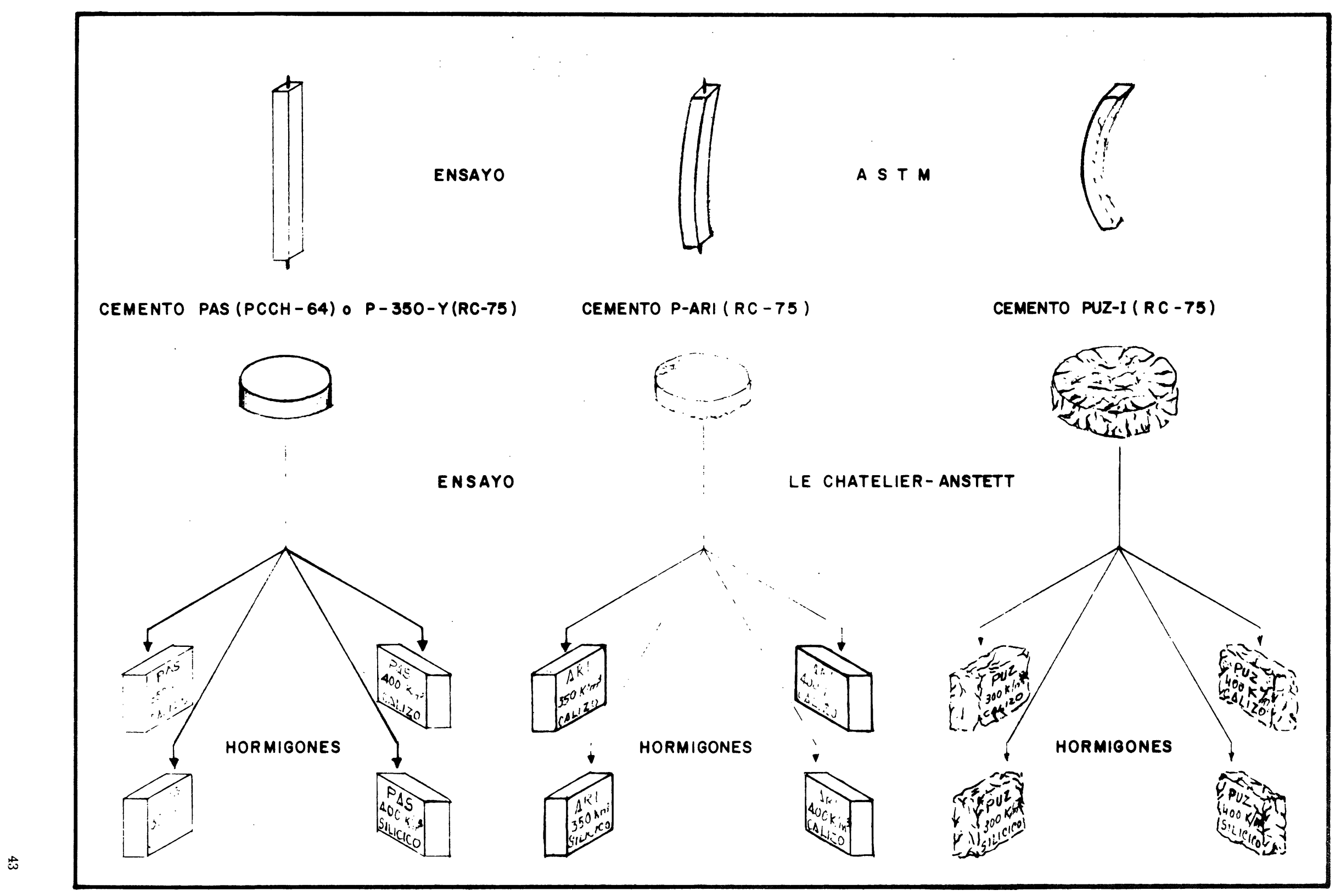




\subsubsection{Consecuencias}

Si se emplea el método de ANSTETT para comparar el comportamiento frente a los sulfatos y en particular frente al yeso, de cementos portland del mismo tipo, pero de distinta clase y/o categoría, normalmente se comportarán mejor los que mejor deben de comportarse en razón de su naturaleza química.

Pero si se emplea dicho método para comparar el comportamiento de un cemento portland de alta resistencia con el de un cemento puzolánico, es posible que, contra lo que cabría esperar, se comporte mejor aquél que éste, como también se ha podido observar recientemente (6). Una razón más de disccriminación del método de ANSTETT, lesiva para los cementos puzolánicos. Estos hechos responden al esquema gráfico (parte superior), el cual es fiel reflejo del caso real (6) observado en el laboratorio.

\section{EL COMPORTAMIENTO DE LOS CEMENTOS EN CONDICIONES PRACTICAS REALES}

Se ha indicado en lo que precede, que en los casos reales de los hormigones en la práctica, los distintos tipos de cementos y los cementos de un mismo tipo pero de distinta clase o categoría, pueden comportarse de modo muy diferente a como se comportan en el ensayo de ANSTETT, y más en consonancia con lo que lógicamente cabría pensar a priori.

Sin embargo, también en estas circunstancias las previsiones apriorísticas pueden y suelen fallar. Tal es el caso (6) de probetas de hormigones con dosificaciones de cemento de 300 y $400 \mathrm{~kg} / \mathrm{m}^{3}$, con tres cementos PUZ-I-350, P-450-ARI (III-ASTM) y P-350-Y (V-ASTM) (7) en cada caso, y con árido silícico de canto rodado y calizo de machaqueo, respectivamente. Las granulometrías y las relaciones agua/cemento $(0,4)$ fueron las mismas, así como la fabricación, el curado y la conservación de las probetas (parte inferior del esquema).

Estas permanecieron a la intemperie durante cuatro años, semienterradas en un terreno yesífero con abundante yeso "espejuelo" a la vista, y cubiertas total y parcialmente según la época del año - salvo en las épocas estivales en que quedaban en seco- por aguas naturales conteniendo sulfatos cálcico y magnésico en disolución, a fuertes concentraciones -la de éste último, de $4 \mathrm{mg} / \mathrm{l}(4.000 \mathrm{ppm})$ -

En tales circunstancias los mecanismos de los ataques al hormigón pueden ser disyuntiva o conjuntamente $-\mathrm{y}$ en este último caso simultáneos o sucesivos-, los siguientes:

1) Formación de ettringita:

$$
\mathrm{C}_{4} \mathrm{AH}_{19}+3 \mathrm{C} \overline{\mathrm{S}}+13 \mathrm{H} \rightarrow \mathrm{CH}+\mathrm{C}_{3} \mathrm{~A}(\mathrm{C} \overline{\mathrm{S}})_{33} \mathrm{H}_{31}
$$

o bien:

$$
\mathrm{C}_{3} \mathrm{AH}_{6}+3 \mathrm{C} \overline{\mathrm{S}}+25 \mathrm{H} \rightarrow \mathrm{C}_{3} \mathrm{~A}(\mathrm{C} \overline{\mathrm{S}})_{33} \mathrm{H}_{31}
$$

2) Formación de brucita:

$$
\overline{\mathrm{MS}}+\mathrm{CH} \rightarrow \mathrm{C} \overline{\mathrm{S}}+\mathrm{MH}
$$


3) Formación (cristalización) de yeso:

$$
\mathrm{C} \overline{\mathrm{S}}+2 \mathrm{H} \rightarrow \mathrm{C} \overline{\mathrm{S}} \mathrm{H}_{2}
$$

Según 1), los hormigones más vulnerables serían los hechos con los cementos de mayor contenido de $\mathrm{C}_{3} \mathrm{~A}$. Por lo tanto, la vulnerabilidad sería creciente en el orden de los cementos

$$
\text { P-350-Y, PUZ-I-350, y P-450-ARI }
$$

o tal vez:

$$
\text { PUZ-I-350 P-350-Y y P-450-ARI }
$$

Según 2), los hormigones más sensibles serían los hechos con los cementos que dan mayor cantidad de cal libre de hidrólisis, es decir, con los de mayor contenido de $\mathrm{C}_{3} \mathrm{~S}$, y/o menor -o nulo- contenido de puzolana. Por consiguiente, la sensibilidad sería creciente en el orden de los cementos

$$
\text { PUZ-I-350, P-350-Y y P-450-ARI }
$$

Según 3), y aparte de la acción propia del yeso, se repetirían los órdenes de vulnerabilidad según 1) y 2). La acción aislada de la cristalización del yeso implicaría el siguente orden creciente de sensibilidad:

$$
\text { P-450-ARI, PUZ-I-350 y P-350-Y }
$$

o tal vez:

$$
\text { P-450-ARI, P-350-Y y PUZ-I-350 }
$$

Es decir, los hormigones con mayor probabilidad de resistir un ataque combinado por sulfato cálcico y por sulfato magnésico, parecen ser, lógicamente, los hechos con los cementos PUZ-I-350 o P-350-Y (en uno u otro orden y casi indistintamente) y, en último término, los hechos con cemento P-450-ARI.

No obstante, los resultados de las observaciones visuales de las probetas al cabo de los cuatro años ponen de relieve los hechos siguientes, muchos de los cuales son los previsibles y no aportan ningún conocimiento nuevo. Otros, en cambio, llaman la atención por su aparente inconsecuencia.

En primer lugar, los hormigones de mayor dosificación de cemento - a igualdad de lo demás - resisten más y mejor, lo cual puede ser debido a una mayor compacidad — menor porosidad, permeabilidad y penetrabilidad por succión capilar-, y a una mayor resistencia mecánica del hormigón, sobre todo en los primeros momentos.

En segundo lugar, los hormigones con árido silícico rodado - y todo lo demás igual- resisten más y mejor que los de árido calizo machacado. Esto puede obedecer, por una parte. a una mayor compacidad, como en el caso anterior; y, por otra parte, a una mayor superficie de contacto en el caso del árido calizo (por la mayor superficie específica de éste), lo cual propicia les defectos de adherencia y las discontinuidades en las zonas de contacto, así como la más fácil penetración del agresivo en profundidad.

En tercer lugar - y esto es lo extraordinario-, dentro de lo ya señalado y a igualdad de todo lo demás, los hormigones de mejor comportamiento son los de cemento P-450-ARI 
(tipo III ASTM), seguidos de los cementos P-350-Y (tipo V ASTM) y, por último, de los cementos PUZ-I-350.

Por lo que respecta a los dos primeros hormigones, la explicación, ya que no puede ser sólo ni mayormente de tipo químico, a no ser que predomine casi en exclusiva el mecanismo 3), ha de ser más bien de tipo físico: una mayor resistencia mecánica del hormigón, en particular en las primeras edades, y una mayor compacidad del hormigón de cemento P-450-ARI, por la mayor categoría resistente de éste y por una plasticidad muy probablemente mayor, al ser el cemento más fino.

En cambio, por lo que respecta a los dos últımos hormigones, la explicación tendría que ser más de tipo químico: el bajo contenido de $\mathrm{C}_{3} \mathrm{~A}$ del primero de ellos (el cemento $\mathrm{P}-350-\mathrm{Y}$ contiene 2 por ciento de $\mathrm{C}_{3} \mathrm{~A}$ ). En este caso falla la explicación de tipo físico, ya que el hormigón de cemento puzolánico debería tener una mayor compacidad por su mayor plasticidad debida a la posible mayor finura del cemento -a no ser que la diferencia de finura entre él y el P-350-Y no fuese notable, y/o que la plasticidad de los hormigones de ambos tampoco difiriese mucho, por lo dicho y por existir diferencias entre las curvas granulométricas de ambos cementos operantes en dicho sentido, dada la relativa dureza de la puzolana del cemento PUZ-I-350.

Por lo que respecta a los hormigones primerc (de P-450-ARI) y último (de PUZ-I-350), vuelve a fallar la explicación química y a primar la física, como en el caso de los dos primeros hormigones: la mayor resistencia mecánica a corto plazo — sobre todo-, y la mayor compacidad por mayor plasticidad, debida a una (posiblemente) mayor finura del cemento, o (más probablemente) a una curva granulométrica más apropiada del mismo.

La explicación del hecho un tanto insólito de que un cemento puzolánico se comporte deficientemente frente al ataque de un terreno y de unas aguas con sulfato cálcico y sulfato magnésico, y sobre todo en comparación con un cemento portland de alta resistencia, así como el hecho de que éste se comporte tan bién o mejor que el cemento portland de bajo contenido de $\mathrm{C}_{3} \mathrm{~A}$, el cual es especialmente resistente al yeso en particular, pero también a los sulfatos en general - por lo menos en mayor medida que los cementos portland ordinarios-, hay que buscarla en los hormigones que se comparan.

Los de cada tipo de árido y de cada dosificación son iguales en todo lo demás, incluso en la relación agua/cemento, que fue de 0,4 en todos los casos. Ahí puede estar el quid de la cuestión y el porqué de las supuestas diferencias de compacidad - e incluso de resistencias mecánicas a corto plazo- entre los hormigones de los distintos cementos que se comparan.

La constancia de relación a/c puede poner aún en mayor inferioridad de condiciones a los hormigones del cemento puzolánico respecto de los otros dos hormigones de los cementos portland, tanto en cuanto a plasticidad y consistencia en estado fresco, como en cuanto a compacidad y a resistencias a corto yi a largo plazo en estado endurecido. Lo primero, porque suele ser normal que los cementos puzolánicos, si la puzolana es adecuada, den hormigones de mayor plasticidad y consistencia que los portland, a igualdad de relación a/c; o de igual plasticidad y consistencia que los portland, con relaciones a/c menores. Lo segundo, porque al comparar los cementos en hormigones con la misma relación a/c, en vez de en hormigones con la misma consistencia en fresco, se está imponiendo a los cementos puzolánicos una condición desventajosa que agrava aún más la propia desventaja de su endurecimiento global más lento y de sus resistencias más bajas, especialmente a corto plazo. 


\section{CONCLUSIONES}

4.1. En el complejo problema de la durabilidad del hormigón, y más concretamente en el de su resistencia a los sulfatos en general y al cálcico y/o magnésico en particular, intervienen tantos factores variables que es muy difícil establecer cuál o cuáles de los posibles mecanismos químicos de ataque a la pasta de cemento y de aspectos físicos inherentes al propio hormigón, son los operantes con preferencia y de forma decisiva en cada caso.

Los factores físicos y mecánicos del hormigón pueden ser tales que, al preponderar en conjunto sobre las acciones y mecanismos químicos, los impidan, dificulten, atenúen o retrasen, o, por el contrario, los provoquen, faciliten, incrementen o aceleren. En ambos casos puede suceder que el comportamiento de los hormigones no corresponda al que se puede y debe esperar de la naturaleza química de los cementos con los que están hechos, de modo que se comporten mejor los hormigones de cementos químicamente menos idóneos, y viceversa.

Sin embargo, de esto no se debe concluir con carácter general que el empleo de los cementos menos aptos sea genéricamente más aconsejable. Al comparar comportamientos de cementos diferentes es preciso hacerlo en condiciones de absoluta y garantizada igualdad de todas las demás circunstancias, sin otras variables que los cementos que se comparan. Pero bien entendido que cuando hay opción entre dos circunstancias - por ejemplo, entre la igualdad de la relación agua/cemento o la igualdad de la consistencia del hormigón-, es necesario escoger aquélla que más convenga a las condiciones reales en la práctica; en este caso, la igualdad de consistencia.

4.2. Por otro lado, cuando se habla de cementos de un determinado tipo o clase, y sobre todo si comportan la presencia de adiciones activas, como es el caso de los cementos de tipo puzolánico —o siderúrgico- (5), no se les debe considerar genéricamente a ultranza, pues entre ellos los puede haber de gran diversidad - y por lo tanto de muy diferente comportamiento-, en función de la naturaleza y proporción de la adición, de la finura y granulometría del cemento, de la absorción y retención de agua y de la plasticidad de sus pastas, de la exudación, rezumado y retracción de las mismas. de la compacidad y resistencia de sus hormigones, etc.

4.3. Por otra parte, entre los métodos de ensayo de laboratorio, y precisamente entre los más drásticos, los hay que no tratan en absoluto plano de igualdad a todos los cementos. Por el contrario, algunos de éstos -y no ciertamente los más idóneos desde un punto de vista químico- pueden resultar beneficiados por algunos métodos, mientras que los cementos más aptos para un determinado tipo de ataque pueden resultar perjudicados.

Tal sucede -entre otros y también con el ASTM-- con el método de ensayo de ANSTETT, el cual puede posponer los cementos puzolánicos a los portland de alta resistencia, endurecimiento ráp:do y elevado calor de hidratación, en cuanto a comportamiento frente a los sulfatos.

En tales casos es muy recomendable, a la hora de comparar en este aspecto cementos puzolánicos con otros cementos, o bien no utilizar el método de ANSTETT, o cuando menos no utilizarlo en exclusiva, o, finalmente, utilizarlo con las modificaciones precisas para poner a los cementos puzolánicos erı condiciones comparables con las de otros cementos, respecto de la aplicabilidad del método y de la correcta interpretación y valoración de sus resultados. 
4.4. Finalmente, si se trata de hacer una recomendación de determinados tipos o clases de cementos para hormigones o elementos estructurales que hayan de resistir determinados tipos de ataque - por ejemplo, para pilotes prefabricados que tengan que estar en contacto con aguas y suelos que contengan yeso y/o sulfato magnésico (1)-, es preciso ofrecer una solución más o menos buena (la mejor posible), sin eludir el problema, porque éste puede ser real, no imaginario —en el ejemplo expuesto el problema es realísimo-. En este caso hay que tener en cuenta que los cementos portland resistentes —específicamente- al yeso — por ejemplo, los P-350-Y (3)—, son, dentro de los portland, también en no escasa medida resistentes a los sulfatos en general, incluido el magnésico. Esto no sólo es apriorístico, sino que está confirmado experimentalmente en la práctica real (6).

\section{R E F E R E N C I A S}

(1) Ministerio de Obras Públicas y Urbanismo: Norma Tecnológica NTE-CPP/1978 "Cimentaciones: Pilotes Prefabricados". BOE núm. 180, pág. 17.804, 29 julio 1978.

(2) Calleja, J. y Garcia de Paredes, P.: "Sobre los Métodos para el Estudio de la Durabilidad de los Conglomerantes Hidráulicos". Materiales de Construcción (IETCC), núm. 137, enero-febrero-marzo 1970.

RILEM Symposium on Concrete Durability, Praga, 1969. Cahier de Recherche (IETCC), núm. 21, 1969.

(3) Calleja, J.: "Código de Buena Práctica para Hormigón resistente a Sulfatos. (Consideraciones sobre Requisitos para los Cementos Portland utilizables)".

Materiales de Construcción (IETCC), núm. 144, octubre-noviembre-diciembre 1971.

"A Code of Good Practice for Concrete resisting Sulphate Attack. (Some Considerations on Requirements for Portland Cements involved)".

Research Working Paper (IETCC), núm. 29, 1973.

(4) Calleja, J.: "Las Nuevas Normas Españolas para Cemento".

Materiales de Construcción (IETCC), núm. 164, octubre-noviembre-diciembre 1976.

INTT - Instituto Nacional de Tecnología Industrial.

Buenos Aires - Argentina, junio 1976.

(5) Calle.Ja, J.: "Cementos Puzolánicos".

Materiales de Construcción (IETCC), núm. 165, enero-febrero-marzo 1977.

INTI - Instituto Nacional de Tecnologia Industrial.

Buenos Aires - Argentina, junio 1976.

(6) Aguanell, M.: Comunicación Privada, 1980.

(7) RC-75: Pliego de Prescripciones Técnicas Generales para la Recepción de Cementos. Ministerio de Obras Públicas y Urbanismo, Secretaria General Técnica, 1975. 